\title{
Influence of Selected Parameters on the Rate Nucleation of Methane Hydrates
}

\author{
Dávid Hečko, ${ }^{1, *}$, Milan Malcho ${ }^{1}$, Pavol Mičko ${ }^{1}$, and Alexander Čaja ${ }^{1}$ \\ ${ }^{1}$ Department of Power Engineering, Faculty of Mechanical Engineering, University of Žilina, Vel'ký \\ diel, 01026 Žilina, Slovakia
}

\begin{abstract}
The demand for energy is growing in the world, and therefore attention has been focused on the use of possible alternative energy sources. For countries with limited access to common hydrocarbon gases, methane hydrates appear to be a potential source of energy. Under certain pressure and temperature conditions, it is also possible to produce hydrates of hydrocarbon gases under laboratory conditions. However, we are currently at a very early stage in the development of methane hydrates. The transition from scientific theory to practical extraction is currently underway. This article describes the influence of selected physical parameters on the nucleation rate of hydrocarbon gas hydrates.
\end{abstract}

\section{Introduction}

Energy storage is an interesting phenomenon today, which tends to move forward. The accumulation of energy in the form of a hydrate is important from the point of view of safety and the possibility of transport to the place of coverage of unexpected energy peaks in the technological industry. Natural gas hydrate is in the solid state, which makes up about $85 \%$ of the water and $15 \%$ of the gas bound in the clathrate lattice. There is also some uncertainty associated with hydrates due to ongoing research and their use as potential sources of income. Insufficient research and development of hydrates has caused that the real application in convection systems of natural gas accumulation is in the phase of theoretical studies. This unconventional energy source is currently destined for the distant path to energy efficiency. Storing energy in a suitable form for safe storage and its subsequent release is a current challenge in processes that use the need to store energy [1].

\section{Chemical equilibrium between solid and gas phase}

This section describes some of the selected parameters needed to determine the hydrate formation model. Particle motion studies in the process of crystallization of hydrocarbon gas hydrates are not sufficiently elucidated and there is a significant difference of opinion, which is actually the initial impetus for the crystallization of the hydrate. The next part of the article is devoted to a general analysis of the criteria for the phase equilibrium of a specific threecomponent system of gas, aqueous solution and crystallized gas hydrate [2].

\footnotetext{
*Corresponding author: david.hecko@,fstroj.uniza.sk
} 


\subsection{Phase equilibrium}

The criteria for phase equilibrium developed by Gibbs are:

1. the temperature and pressure of the phases are the same,

2. each of the components has a chemical potential at each stage,

3. the global Gibbs energy is minimal.

These criteria relate to the phase balance of hydrates and form the basic models for performing mathematical calculations of hydrate balance. Most phase equilibrium calculations transition from chemical potentials to a property related to its partial pressure, which expresses the tendency to escape or expand. However, hydrate calculations are usually performed on the basis of simple chemical potentials. Minimizing free energy is important when calculating hydrates. The stable hydrate phase (type I, II or even $\mathrm{H}$ ) is the one that leads to a minimum in Gibbs free energy. Therefore, simply meeting the first two criteria is not enough to solve the hydrate problem. From a thermodynamic point of view, the hydrate formation process can be modeled to be carried out in two steps. The first step starts from clean water to an empty hydrate cage. This first step is hypothetical, but useful for calculation purposes. The second step is to fill the hydrate grid. The process is as follows:

Pure water $(\alpha) \rightarrow$ empty hydrate grid $(\beta) \rightarrow$ filled hydrate grid $(H)$. The change in chemical potential for this process is reported as:

$$
\mu^{H}-\mu^{\alpha}=\left(\mu^{H}-\mu^{\beta}\right)+\left(\mu^{\beta}-\mu^{\alpha}\right)
$$

where $\mu$ is the chemical potential and the superscripts refer to different phases. The first term after the equal sign represents the stabilization of the hydrate lattice. It is a variation in the models used to estimate the conditions that separate the different models.

The second condition represents a phase change for water and can be calculated by conventional thermodynamic means. This condition is calculated as follows:

$$
\frac{\mu^{\beta}-\mu^{\alpha}}{R T}=\frac{\Delta \mu(T, P)}{R T}=\frac{\Delta \mu\left(T_{o}, P_{o}\right)}{R T_{o}}-\int_{T_{o}}^{T} \frac{\Delta H}{R T^{2}} d T+\int_{P_{o}}^{P} \frac{\Delta v}{R \bar{T}} d P,
$$

where $R$ is the universal gas constant, $T$ is the absolute temperature, $P$ is the pressure, $H$ is the enthalpy, $v$ is the molar volume, the index 0 represents the reference state and the terms $\mathrm{D}$ represent the change from pure water (either liquid or ice) to the hydration phase (either of type I or II). The line above the temperature in the last component of the relationship indicates that this is the average temperature.

\subsection{Van der Waals and Platteuwa method}

The first model for calculating hydrate formation was that of Van der Waals and Platteeuw (1959). They presented their model as a statistical model of hydrate formation. The concentration of non-aqueous species in the hydrate was treated in a manner similar to gas adsorption to a solid. For one guest molecule, this term is evaluated as follows:

$$
\mu^{H}-\mu^{\beta}=R T \sum_{i} v_{i} \ln \left(1-Y_{i}\right),
$$


where $v_{i}$ is the number of type I cavities and $\mathrm{Y}$ is the probability function. The probability $\mathrm{Y}$ that a type I cavity is occupied by a guest is given by:

$$
Y_{i}=\frac{c_{i} P}{1+c_{i} P}
$$

where $c_{i}$ in this equation is a function of the host molecule and the cage and $P$ is the pressure. Although it is not clear from this definition $c_{i}$ are also a function of temperature. A simple test, because ethane occupies only small crystal cages of type I hydrate, then $c_{i}$ for ethane and for small cages is zero. On the other hand, methane, which is also a former type I, occupies all large and small cages. Therefore, both values of $c_{i}$ in the formula for this component are non-zero.

\subsection{Van der Waals and Platteuwa method}

The findings of the original van der Waals and Platteeuw methods (1959) provided a good basis for performing hydrate calculations, but were not accurate enough for technical calculations. One of the first models with the necessary accuracy for technical calculations was the Parrish and Prausnitz model (1972). There are two major differences between the original van der Waals and Platteeuw (1959) model and the model proposed by Parrish and Prausnitz (1972). First, they extended the model to hydrate mixtures. This is expressed as follows:

$$
\mu^{H}-\mu^{\beta}=R T \sum_{i} v_{i} \ln \left(1-\sum_{K} Y_{K i}\right)
$$

where the most noticeable difference can be seen in the operator of the sum used over all components of the probability function.

$$
Y_{K i}=\frac{c_{i} P_{K}}{1+\sum_{j} c_{i j} P_{j}},
$$

where $c_{i}$ is the sum of the components of the guest molecules and PK followed by the subscript is the partial pressure for the component. Thus, the presence of another guest molecule reduces the likelihood that a given guest molecule can enter the hydrate lattice. Second, Parrish and Prausnitz (1972) replaced partial pressure with fugacity. There is no simple definition for fugacity thermodynamics. Common definitions given in thermodynamic textbooks are derived from the chemical potential of partial free enthalpy in thermodynamic processes. For our purposes, we can consider fugacity as a "corrected" pressure, which is assessed as not ideal, so its replacement results in the following relationship:

$$
Y_{K i}=\frac{c_{i} \hat{f}_{I}}{1+\sum_{j} c_{i j} \hat{f}_{j}},
$$

where $\hat{f}_{I}$ is the fugacity of component $\mathrm{I}$ in the gas mixture. This allowed their model to take into account the uncertainties in the gas phase, and thus it was possible to extend the model to higher pressures. In addition, some of the parameters in the model have been adjusted to 
reflect the change from fugacity pressures, thereby improving the overall fit of the model. This means that this model has a different file $c_{i}$, which had to be changed from a pressure model to a fugacity model [3].

\section{Model study of hydrate formation}

The hydrate formation process was based on a patented system of two high-pressure vessels. In the first vessel, the methane is hydrated and then pumped to the second vessel, where the process of capture and dehydration takes place, i.e. the removal of excess water from the mixture $[4,9]$.

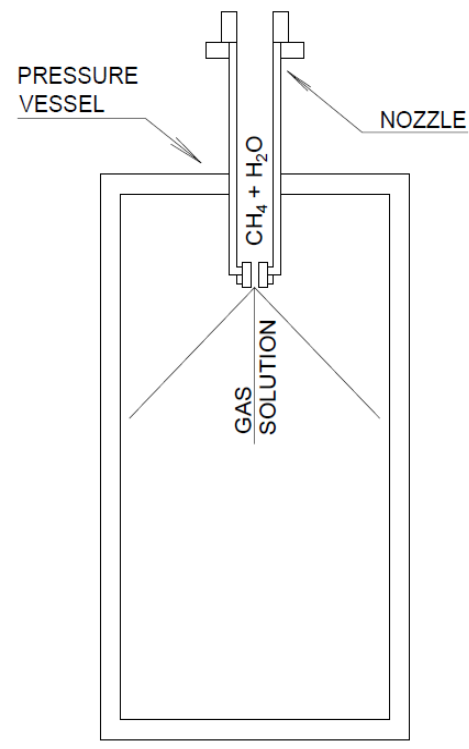

Fig. 1. Schematic diagram spraying water and methane into a pressure vessel.

The production of methane hydrates is conditioned by the formation of contacts between the liquid and the gas. This process is schematically illustrated in Figure 1. Therefore, the method of injecting water droplets into the gas continuous phase was chosen. Injection of water into the gas at the stage of their interaction causes the formation of hydrates. This phenomenon takes place in two phases: nucleation and embryo growth. During nucleation, methane gas disperses in the aqueous component. Dissolved methane in water forms hydrate nuclei and crystallizes. During the growth phase, the germs formed grow in size or aggregate. Crystallization of the hydrate is considered successful in achieving the required pressure and temperature conditions for the time necessary to form the clathrate structure [5-7]. The basic parameters characterizing the hydrates can be seen in the table 1.

Table 1. Parameters characterizing hydrate formation.

\begin{tabular}{|c|c|c|c|c|c|c|c|}
\hline Gas & Hydrate structure & $n_{w}$ & $n_{g}$ & $v_{h}\left[\mathrm{~nm}^{-3}\right]$ & $-\Delta v_{e}\left[\mathrm{~nm}^{-3}\right]$ & $-b\left[\mathrm{~nm}^{3}\right]$ & $v_{e f}\left[\mathrm{~nm}^{3}\right]$ \\
\hline $\mathrm{CH}_{4}$ & sI & $23 / 4$ & 8 & 0.216 & 0.044 & 0.086 & 0.130 \\
\hline
\end{tabular}


Hydrate crystal structurea and values of hydration number $n_{w}$ of stoichiometric hydrate crystal, number $n_{g}$ of gas molecules per unit cell of stoichiometric hydrate crystala, volume $v_{h}$ of hydrate building unit, volume difference $\Delta v_{e}$, second virial coefficient $b$ (at $\mathrm{T}=273.2 \mathrm{~K}$ ) and effective volume $v_{e f}$.

According to the above-mentioned relationships, the impulse for the formation of a new phase is the difference between the chemical potentials of the old and the new phase. This difference is called supersaturation and is commonly referred to as $\Delta \mu$. The phase equilibrium between the solution and the hydrate is characterized by $\Delta \mu=0$. At the selected temperature $T$, the supersaturation becomes zero at the so-called equilibrium pressure $P_{e}$.

$$
\begin{gathered}
\Delta \mu=k T \ln [\phi(P, T) P]+\mu_{g g, 0}(T)+n_{w}(P, T) \mu_{w, 0}(T)-\mu_{h, 0}(T)+n_{w}(P, T) \int 0 P v_{w}\left(P^{\prime}, T\right) \\
d P^{\prime}-\int 0 P v_{w}\left(P^{\prime}, T\right) d P^{\prime}-\int 0 P v_{h}\left(P^{\prime}, T\right) d P^{\prime},
\end{gathered}
$$

where $P$ - is the pressure in the reactor and $T$ - the temperature in the reactor.

When calculating the equilibrium pressure in equation (8), we replace the pressure in the reactor $P$ with the equilibrium pressure $P_{e}$, Which allows us to remove the reference chemical potentials $\mu_{g g, 0}$ and $\mu_{h, 0}$. The expression will take the following form:

$$
\begin{gathered}
\Delta \mu=k T \ln \left[\phi(P, T) P / \phi\left(P_{e}, T\right) P_{e}\right]+\left[n_{w}(P, T)-n_{w}\left(P_{e}, T\right)\right] \mu_{w, 0}(T)+ \\
n_{w}(P, T) \int 0 P v_{w}\left(P^{\prime}, T\right) d P^{\prime}-\int 0 P_{e} v_{w}\left(P^{\prime}, T\right) d P^{\prime}-\int P_{e} P v_{h}\left(P^{\prime}, T\right) d P^{\prime}
\end{gathered}
$$

Assuming that water and natural gas are in chemical equilibrium in the reactor, this general formula can be simplified by approximation to a more acceptable form:

$$
\Delta \mu=k \operatorname{Tln}\left(P / P_{e}\right)+v_{e f}\left(P / P_{e}\right)
$$

This approximation is applicable for a pressure close to the equilibrium pressure in our case it corresponds to the required reactor pressure of $10 \mathrm{MPa}$, where the calculation of equations (9) and (10) has results very similar with a negligible deviation. Significant deviation occurs only at pressures higher than $15 \mathrm{MPa}$. So it follows that relationship number 10 is applicable to our case $[2,8]$.

\section{Application of theoretical knowledge to specific issues}

The equilibrium pressure Pe, was calculated from the previous expression and indicates that the water-gas solution is in the equilibrium phase. The calculation was performed in isothermal mode. The dependence of pressure can be seen in the Figure 2. 


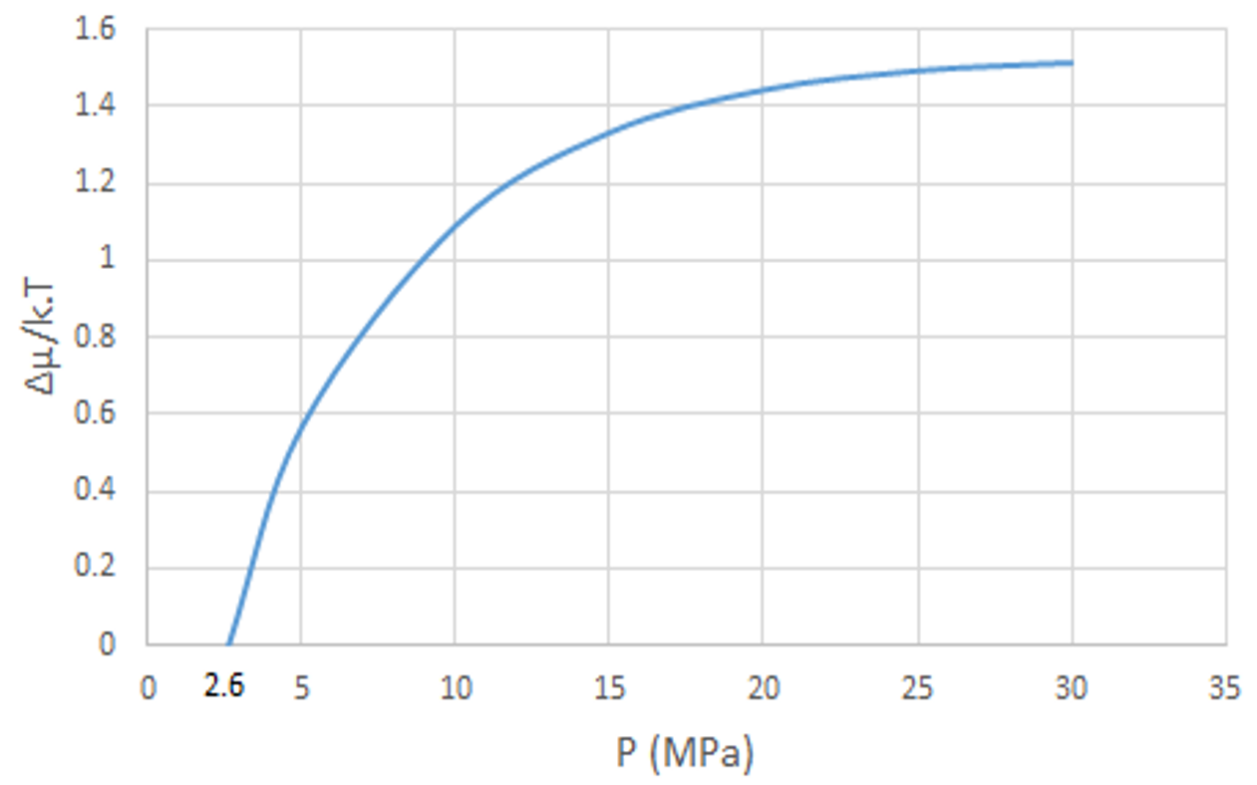

Fig. 2. Pressure dependence of supersaturation for crystallization of methane hydrate.

The calculation of the supersaturation value at the required pressure in the reactor pressurized to $10 \mathrm{MPa}$ and at a temperature of $273.15 \mathrm{~K}$ was continued. The supersaturation value $\Delta \mu$ is given in the following Table 2.

Table 2. Parameters characterizing equilibrium phase.

\begin{tabular}{|c|c|c|c|c|}
\hline Definition & Designation & Units & Entered & Calculated \\
\hline Supersaturation & $\Delta \mu$ & $\mathrm{J}$ & & $4.12^{-21}$ \\
\hline The second viral coefficient & $b$ & $\mathrm{~m}^{3}$ & $-8.60^{-29}$ & \\
\hline Volume difference & $\Delta v_{e}$ & $\mathrm{~m}^{3}$ & $-4.40^{-29}$ & \\
\hline Effective volume & $v_{e f}$ & $\mathrm{~m}^{3}$ & & $-1.30^{-28}$ \\
\hline Equilibrium pressure & $P_{e}$ & $\mathrm{~Pa}$ & 2600000 & \\
\hline Boltzmann's Constant & $k$ & $\mathrm{~J} . \mathrm{K}^{-1}$ & $1.3806^{-23}$ & \\
\hline Temperature & $T$ & $\mathrm{~K}$ & 273.15 & \\
\hline Pressure & $P$ & $\mathrm{~Pa}$ & 10000000 & \\
\hline
\end{tabular}

\section{Conclusion}

This paper analyzes the crystallization process and the growth rate of natural gas hydrates based on the supersaturation of the water and gas solution in the phase equilibrium state of the three-component water, gas and hydrate system. The theoretical analysis of the issue gives a derived formula adjusted by approximation to a usable shape for our experimental equipment. It follows from the relationship that the primary impulse for the crystallization of 
hydrates is the supersaturation $\Delta \mu$, which depends on the actual concentration of the gas in the solution and is sensitive to the specific conditions of dissolution of the gas into the aqueous phase [6].

Work on article has been financially supported by the project VEGA-1/0738 / 18 „Optimization of energy inputs for the rapid generation of natural gas and biomethane hydrates for the accumulation of high potential primary energy"

\section{References}

1. P. Pilat, M. Patsch, J. Jandacka, EFM11, 25 (2012)

2. D. Kashchiev, A. Firoozabadi, Journal of Crystal Growth 241, 220-230 (2002)

3. J. Carroll, Natural gas hydrates: A guide for en-gineers (Elservier Science, Burlington, 2014)

4. A. Kapjor, T. Gressak, J. Huzvar, M. Vantuch, AIP Conference Proceedings, 1608, 103107 (2014)

5. J. Huzvar, J. Jandacka, P. Pilat, EFM08, 58-60 (2008)

6. J. Jandačka, M. Holubčík, Mobile Networks \& Applications, 25/3, 904-912 (2020)

7. J. Jandačka, M. Holubcik, S. Papucik, R. Nosek, Acta Montanistica Slovaca, 17, 283-289 (2012)

8. R. Nosek, M. Holubčík, Acta Facultatis Xylologiae Zvolen, 58/1, 105-112

9. J. Siažik, M. Malcho, R. Lenhard, EPJ Web of Conferences 143, 02106 (2017)

10. M. Malcho, R. Lenhard, K. Kaduchova, AIP Conference Proceedings 2000, 020014 (2018) 